Supplementary Material to Accompany:

\title{
Group IV Metal Complexes Catalyzed the Intermolecular Hydroamination of Methylenecyclopropane
}

Elena Smolensky, Moshe Kapon and Moris S. Eisen*

Schulich Faculty of Chemistry and Institute of Catalysis Science and Technology, Technion-Israel Institute of Technology, Haifa, 32000, Israel. 


\section{Mathematical Derivation}

rate $=-\frac{d[\mathrm{PhMCP}]}{d t}=\frac{d[9]}{d t}=k_{4}[8]$

Steady state assumption for $3,6,7,8$

$$
\begin{aligned}
& \frac{d[8]}{d t}=k_{3}[\text { amine }][7]-k_{4}[8]=0 \quad \Leftrightarrow[7]=\frac{k_{4}[8]}{k_{3}[\text { amine }]} \\
& \frac{d[7]}{d t}=k_{2}[6]-k_{3}[\text { amine }][7]=0 \quad \Leftrightarrow[6]=\frac{k_{3}[\text { amine }][7]}{k_{2}}=\frac{k_{3}[\text { amine }] k_{4}[8]}{k_{2} k_{3}[\text { amine }]}=\frac{k_{4}[8]}{k_{2}} \\
& \frac{d[6]}{d t}=k_{1}[\mathrm{PhMCP}][3]-k_{-1}[6]-k_{2}[6]=0 \quad \Leftrightarrow[3]=\frac{\left(k_{-1}+k_{2}\right)[6]}{k_{1}[\mathrm{PhMCP}]}=\frac{\left(k_{-1}+k_{2}\right)}{k_{1} k_{2}[\mathrm{PhMCP}]} k_{4}[8] \\
& \frac{d[6]}{d t}=k_{4}[8]+k_{-1}[6]-k_{1}[\mathrm{PhMCP}][3]+k_{5}[4]^{1 / 2}+k_{6}[2]-k_{-5}[3]-k_{-6}[\text { amine }][3]=0 \\
& \Leftrightarrow k_{4}[8]+\frac{k_{-1}}{k_{2}} k_{4}[8]-\frac{k_{1}[\mathrm{PhMCP}]\left(k_{-1}+k_{2}\right)}{k_{1} k_{2}[\mathrm{PhMCP}]} k_{4}[8]+k_{5}[4]^{1 / 2}+k_{6}[2]-\frac{k_{-5}\left(k_{-1}+k_{2}\right)}{k_{1} k_{2}[\mathrm{PhMCP}]} k_{4}[8] \\
& -\frac{k_{-6}[\mathrm{amine}]\left(k_{-1}+k_{2}\right)}{k_{1} k_{2}[\mathrm{PhMCP}]} k_{4}[8]=0 \\
& \Leftrightarrow k_{4}[8]\left(\frac{\left(k_{-6}[\mathrm{amine}]+k_{-5}\right)\left(k_{-1}+k_{2}\right)}{k_{1} k_{2}[\mathrm{PhMCP}]}=k_{5}[4]^{1 / 2}+k_{6}[2]\right. \\
& \Leftrightarrow k_{4}[8]=\frac{k_{1} k_{2}[\mathrm{PhMCP}]\left(k_{5}[4]^{1 / 2}+k_{6}[2]\right)}{\left(k_{-6}[\mathrm{amine}]+k_{-5}\right)\left(k_{-1}+k_{2}\right)} \\
& \text { rate }=\frac{d[9]}{d t}=k_{4}[8]=\frac{k_{1} k_{2}\left(k_{5}[4]^{1 / 2}+k_{6}[2]\right)(\mathrm{PhMCP}]}{\left(k_{-1}+k_{2}\right)\left(k_{-6}[\mathrm{amine}]+k_{-5}\right)} \\
& \text { rate }=-\frac{d[\mathrm{PhMCP}]}{d t}=\frac{k_{1} k_{2}\left(k_{5}[4]^{1 / 2}+k_{6}[2]\right)(\mathrm{PhMCP}]}{\left(k_{-1}+k_{2}\right)\left(k_{-6}[\mathrm{amine}]+k_{-5}\right)}
\end{aligned}
$$


Table 1. Crystal Data Collection for Complex 13.

\begin{tabular}{ll}
\hline Empirical formula & $\mathrm{C}_{69} \mathrm{H}_{68} \mathrm{~N}_{7} \mathrm{P}_{3} \mathrm{Zr}$ \\
Formula weight & 1179.43 \\
Temperature & $230.0(1) \mathrm{K}$ \\
Wavelength & $0.71073 \AA$ \\
Crystal system, space group & orthorhombic, Pbca \\
Unit cell dimensions & $\mathrm{a}=20.6130(6) \AA$ alpha $=90 \mathrm{deg}$. \\
& $\mathrm{b}=21.2610(6) \AA$ beta $=90 \mathrm{deg}$. \\
& $\mathrm{c}=27.9210(10) \AA$ gamma $=90 \mathrm{deg}$. \\
Volume & $12236.5(7) \AA^{3}$ \\
Z, Calculated density & $8,1.280 \mathrm{Mg} / \mathrm{m}^{3}$ \\
Absorption coefficient & $0.306 \mathrm{~mm} \mathrm{~m}^{-1}$ \\
F(000) & 4928 \\
Crystal size & $0.30 \mathrm{x} 0.28 \mathrm{x} 0.12 \mathrm{~mm}$ \\
Theta range for data collection & 1.56 to $22.99 \mathrm{deg}$. \\
Limiting indices & $-22<=\mathrm{h}<=22,-23<=\mathrm{k}<=23,-30<=1<=30$ \\
Reflections collected / unique & $15766 / 8374[\mathrm{R}(\mathrm{int})=0.1090]$ \\
Completeness to theta $=22.99$ & $98.30 \%$ \\
Max. and min. transmission & 0.9643 and 0.9139 \\
Refinement method & Full-matrix least-squares on $\mathrm{F}^{\wedge} 2$ \\
Data / restraints / parameters & $8374 / 0 / 706$ \\
Goodness-of-fit on $\mathrm{F}^{\wedge} 2$ & 0.881 \\
Final R indices [I $>2$ sigma(I)] & $\mathrm{R} 1=0.0563, \mathrm{wR} 2=0.1136$ \\
R indices (all data) & $\mathrm{R} 1=0.1475, \mathrm{wR} 2=0.1334$ \\
Largest diff. peak and hole & 0.360 and $-0.335 \mathrm{e} . \AA^{-3}$ \\
\hline
\end{tabular}


Table 2. Atomic coordinates $\left(\mathrm{x} 10^{4}\right)$ and equivalent isotropic displacement parameters $\left(\AA^{2} \mathrm{x}\right.$ $10^{3}$ ) for complex 13. $U(\mathrm{eq})$ is defined as one third of the trace of the orthogonalized Uij tensor.

\begin{tabular}{|c|c|c|c|c|}
\hline & $\mathrm{X}$ & $\mathrm{y}$ & $\mathrm{z}$ & $\mathrm{U}(\mathrm{eq})$ \\
\hline $\operatorname{Zr}(1)$ & $4586(1)$ & $3209(1)$ & $684(1)$ & $34(1)$ \\
\hline $\mathrm{P}(1)$ & $4268(1)$ & $2400(1)$ & $1804(1)$ & $48(1)$ \\
\hline $\mathrm{P}(2)$ & $3800(1)$ & $4435(1)$ & $-12(1)$ & $40(1)$ \\
\hline $\mathrm{P}(3)$ & $4359(1)$ & $1708(1)$ & $97(1)$ & $42(1)$ \\
\hline $\mathrm{N}(1)$ & $5569(2)$ & $2693(2)$ & $835(2)$ & $41(1)$ \\
\hline $\mathrm{N}(2)$ & $4726(2)$ & $2578(2)$ & $1318(2)$ & $36(1)$ \\
\hline $\mathrm{N}(3)$ & $3484(2)$ & $2951(2)$ & $754(2)$ & $39(1)$ \\
\hline $\mathrm{N}(4)$ & $3799(2)$ & $3774(2)$ & $331(2)$ & $36(1)$ \\
\hline $\mathrm{N}(5)$ & $5157(2)$ & $3352(2)$ & $-26(2)$ & $34(1)$ \\
\hline $\mathrm{N}(6)$ & $4596(2)$ & $2471(2)$ & $104(2)$ & $39(1)$ \\
\hline $\mathrm{N}(7)$ & $5053(2)$ & $3944(2)$ & $985(2)$ & $42(1)$ \\
\hline$C(1)$ & $6179(3)$ & 2593(3) & $681(3)$ & $52(2)$ \\
\hline$C(2)$ & $6607(3)$ & $2223(3)$ & $926(3)$ & $59(2)$ \\
\hline$C(3)$ & $6405(3)$ & 1958(3) & $1347(3)$ & $59(2)$ \\
\hline$C(4)$ & $5785(3)$ & 2051(3) & $1514(3)$ & $50(2)$ \\
\hline$C(5)$ & $5362(3)$ & $2426(2)$ & $1243(2)$ & $39(2)$ \\
\hline$C(6)$ & $4436(3)$ & $1557(3)$ & $1910(3)$ & $55(2)$ \\
\hline$C(7)$ & $4379(3)$ & 1168(3) & $1515(3)$ & $69(2)$ \\
\hline$C(8)$ & $4518(5)$ & $517(4)$ & $1556(4)$ & $100(3)$ \\
\hline$C(9)$ & $4685(6)$ & $272(4)$ & $1980(5)$ & $133(5)$ \\
\hline$C(10)$ & $4721(5)$ & $644(4)$ & 2381(4) & $113(4)$ \\
\hline $\mathrm{C}(11)$ & $4606(4)$ & $1290(3)$ & $2342(3)$ & $80(2)$ \\
\hline$C(12)$ & $4663(3)$ & $2777(3)$ & $2319(2)$ & $43(2)$ \\
\hline$C(13)$ & $5206(3)$ & $3160(3)$ & $2266(2)$ & $48(2)$ \\
\hline$C(14)$ & $5444(4)$ & $3479(3)$ & $2660(3)$ & $61(2)$ \\
\hline$C(15)$ & $5180(4)$ & $3425(3)$ & $3100(3)$ & $70(2)$ \\
\hline$C(16)$ & $4638(4)$ & $3057(4)$ & $3162(3)$ & $75(2)$ \\
\hline
\end{tabular}




\begin{tabular}{|c|c|c|c|c|}
\hline$C(17)$ & $4375(3)$ & $2732(3)$ & $2772(3)$ & $66(2)$ \\
\hline$C(18)$ & $3062(3)$ & $2512(3)$ & $904(2)$ & $50(2)$ \\
\hline$C(19)$ & $2437(3)$ & 2481(3) & $744(3)$ & $52(2)$ \\
\hline$C(20)$ & $2232(3)$ & $2928(3)$ & $421(3)$ & $52(2)$ \\
\hline $\mathrm{C}(21)$ & $2657(3)$ & $3379(3)$ & $261(2)$ & $45(2)$ \\
\hline$C(22)$ & $3289(3)$ & $3391(2)$ & $434(2)$ & $37(2)$ \\
\hline$C(23)$ & $3140(3)$ & $4933(2)$ & $220(2)$ & $36(2)$ \\
\hline$C(24)$ & $2872(3)$ & $4840(3)$ & $666(3)$ & $49(2)$ \\
\hline$C(25)$ & $2423(3)$ & $5246(3)$ & $858(3)$ & $56(2)$ \\
\hline$C(26)$ & $2235(3)$ & $5777(3)$ & $605(3)$ & $58(2)$ \\
\hline$C(27)$ & $2506(3)$ & $5882(3)$ & $160(3)$ & $58(2)$ \\
\hline$C(28)$ & 2959(3) & $5464(3)$ & $-22(3)$ & $47(2)$ \\
\hline C(29) & $3484(3)$ & 4197(3) & $-604(2)$ & $43(2)$ \\
\hline $\mathrm{C}(30)$ & $2895(3)$ & $4337(3)$ & $-807(3)$ & $51(2)$ \\
\hline$C(31)$ & $2739(4)$ & $4186(3)$ & $-1271(3)$ & $66(2)$ \\
\hline$C(32)$ & $3170(5)$ & $3874(3)$ & $-1544(3)$ & $83(3)$ \\
\hline $\mathrm{C}(33)$ & $3772(5)$ & $3716(4)$ & $-1362(3)$ & $80(3)$ \\
\hline$C(34)$ & $3923(4)$ & $3877(3)$ & $-897(3)$ & $63(2)$ \\
\hline$C(35)$ & $5546(3)$ & $3740(3)$ & $-269(3)$ & $46(2)$ \\
\hline$C(36)$ & $5817(3)$ & $3565(3)$ & $-693(3)$ & $54(2)$ \\
\hline$C(37)$ & $5675(3)$ & $2980(3)$ & $-889(2)$ & $50(2)$ \\
\hline$C(38)$ & $5255(3)$ & $2584(3)$ & $-642(3)$ & $48(2)$ \\
\hline C(39) & $5002(3)$ & $2775(3)$ & $-205(2)$ & $36(2)$ \\
\hline$C(40)$ & $3915(3)$ & $1607(3)$ & $-462(2)$ & $41(2)$ \\
\hline$C(41)$ & $3610(3)$ & $2115(3)$ & $-687(3)$ & $55(2)$ \\
\hline$C(42)$ & 3191(3) & 2026(4) & $-1072(3)$ & $70(2)$ \\
\hline $\mathrm{C}(43)$ & $3066(3)$ & $1430(4)$ & $-1240(3)$ & $79(3)$ \\
\hline $\mathrm{C}(44)$ & $3364(4)$ & $933(4)$ & $-1014(3)$ & $77(2)$ \\
\hline$C(45)$ & $3776(3)$ & $1008(3)$ & $-626(3)$ & $56(2)$ \\
\hline$C(46)$ & $5102(3)$ & $1241(2)$ & $-11(3)$ & $42(2)$ \\
\hline$C(47)$ & $5334(3)$ & $999(3)$ & $-439(3)$ & $58(2)$ \\
\hline
\end{tabular}




\begin{tabular}{lllll}
$\mathrm{C}(48)$ & $5903(4)$ & $655(3)$ & $-461(3)$ & $65(2)$ \\
$\mathrm{C}(49)$ & $6254(4)$ & $545(3)$ & $-54(3)$ & $65(2)$ \\
$\mathrm{C}(50)$ & $6042(3)$ & $771(3)$ & $376(3)$ & $66(2)$ \\
$\mathrm{C}(51)$ & $5477(3)$ & $1119(3)$ & $396(3)$ & $53(2)$ \\
$\mathrm{C}(52)$ & $4608(3)$ & $4218(2)$ & $1326(2)$ & $45(2)$ \\
$\mathrm{C}(53)$ & $4457(3)$ & $4917(2)$ & $1237(3)$ & $70(2)$ \\
$\mathrm{C}(54)$ & $5690(3)$ & $4237(3)$ & $946(3)$ & $56(2)$ \\
$\mathrm{C}(55)$ & $6085(3)$ & $4244(3)$ & $1413(3)$ & $73(2)$ \\
$\mathrm{C}(56)$ & $2570(4)$ & $1875(4)$ & $2216(3)$ & $82(3)$ \\
$\mathrm{C}(57)$ & $2498(4)$ & $1344(4)$ & $1967(4)$ & $90(3)$ \\
$\mathrm{C}(58)$ & $1896(5)$ & $1107(4)$ & $1864(3)$ & $81(3)$ \\
$\mathrm{C}(59)$ & $1361(4)$ & $1424(4)$ & $2020(3)$ & $74(2)$ \\
$\mathrm{C}(60)$ & $1434(4)$ & $1968(4)$ & $2284(3)$ & $73(2)$ \\
$\mathrm{C}(61)$ & $2045(4)$ & $2191(4)$ & $2384(3)$ & $66(2)$ \\
$\mathrm{C}(62)$ & $2140(4)$ & $2781(4)$ & $2686(3)$ & $109(3)$ \\
$\mathrm{C}(63)$ & $3419(14)$ & $4782(12)$ & $2771(11)$ & $172(11)$ \\
$\mathrm{C}(64)$ & $3324(11)$ & $4018(9)$ & $2175(9)$ & $94(7)$ \\
$\mathrm{C}(65)$ & $1970(7)$ & $4303(7)$ & $1921(6)$ & $171(5)$ \\
$\mathrm{C}(66)$ & $2121(10)$ & $3803(9)$ & $1606(8)$ & $105(7)$ \\
$\mathrm{C}(67)$ & $2829(14)$ & $3753(11)$ & $1788(10)$ & $117(9)$ \\
$\mathrm{C}(68)$ & $3084(7)$ & $4430(7)$ & $2397(6)$ & $163(5)$ \\
$\mathrm{C}(69)$ & $2382(11)$ & $4698(8)$ & $2367(7)$ & $69(5)$ \\
$\mathrm{C}(70)$ & $3047(8)$ & $3934(7)$ & $1994(7)$ & $43(4)$ \\
$\mathrm{C}(71)$ & $2000(8)$ & $4619(7)$ & $2240(6)$ & $55(4)$ \\
$\mathrm{C}(72)$ & $2621(10)$ & $4802(8)$ & $2607(8)$ & $84(6)$ \\
$\mathrm{C}(73)$ & $2447(10)$ & $3971(8)$ & $1826(7)$ & $72(5)$ \\
$\mathrm{C}(74)$ & $3719(10)$ & $4387(9)$ & $2575(9)$ & $118(7)$ \\
\hline
\end{tabular}


Table 3. Bond lengths [A] and angles [deg] for complex 13.

\begin{tabular}{|c|c|}
\hline Bonds and Angles & Lengths $[\AA]$ and Angles [deg] \\
\hline $\mathrm{Zr}(1)-\mathrm{N}(7)$ & $2.018(4)$ \\
\hline $\mathrm{Zr}(1)-\mathrm{N}(2)$ & $2.240(5)$ \\
\hline $\mathrm{Zr}(1)-\mathrm{N}(4)$ & $2.247(4)$ \\
\hline $\operatorname{Zr}(1)-\mathrm{N}(6)$ & $2.253(5)$ \\
\hline $\operatorname{Zr}(1)-\mathrm{N}(5)$ & $2.324(5)$ \\
\hline $\mathrm{Zr}(1)-\mathrm{N}(1)$ & $2.342(5)$ \\
\hline $\mathrm{Zr}(1)-\mathrm{N}(3)$ & $2.345(5)$ \\
\hline $\operatorname{Zr}(1)-C(39)$ & $2.783(7)$ \\
\hline $\operatorname{Zr}(1)-C(5)$ & $2.786(6)$ \\
\hline $\operatorname{Zr}(1)-C(22)$ & $2.790(6)$ \\
\hline $\mathrm{Zr}(1)-\mathrm{C}(52)$ & $2.797(6)$ \\
\hline $\mathrm{P}(1)-\mathrm{N}(2)$ & $1.695(5)$ \\
\hline$P(1)-C(12)$ & $1.839(7)$ \\
\hline$P(1)-C(6)$ & $1.849(7)$ \\
\hline $\mathrm{P}(2)-\mathrm{N}(4)$ & $1.701(4)$ \\
\hline$P(2)-C(23)$ & $1.842(6)$ \\
\hline $\mathrm{P}(2)-\mathrm{C}(29)$ & $1.845(7)$ \\
\hline $\mathrm{P}(3)-\mathrm{N}(6)$ & $1.694(4)$ \\
\hline$P(3)-C(40)$ & $1.821(6)$ \\
\hline$P(3)-C(46)$ & $1.852(6)$ \\
\hline $\mathrm{N}(1)-\mathrm{C}(5)$ & $1.343(7)$ \\
\hline $\mathrm{N}(1)-\mathrm{C}(1)$ & $1.347(7)$ \\
\hline $\mathrm{N}(2)-\mathrm{C}(5)$ & $1.365(7)$ \\
\hline $\mathrm{N}(3)-\mathrm{C}(18)$ & $1.343(7)$ \\
\hline $\mathrm{N}(3)-\mathrm{C}(22)$ & $1.354(7)$ \\
\hline $\mathrm{N}(4)-\mathrm{C}(22)$ & $1.360(6)$ \\
\hline $\mathrm{N}(5)-\mathrm{C}(35)$ & $1.336(7)$ \\
\hline $\mathrm{N}(5)-\mathrm{C}(39)$ & $1.364(7)$ \\
\hline
\end{tabular}




$\begin{array}{ll}\mathrm{N}(6)-\mathrm{C}(39) & 1.365(7) \\ \mathrm{N}(7)-\mathrm{C}(52) & 1.446(7) \\ \mathrm{N}(7)-\mathrm{C}(54) & 1.458(7) \\ \mathrm{C}(1)-\mathrm{C}(2) & 1.365(9) \\ \mathrm{C}(1)-\mathrm{H}(1) & 0.93 \\ \mathrm{C}(2)-\mathrm{C}(3) & 1.368(9) \\ \mathrm{C}(2)-\mathrm{H}(2) & 0.9299 \\ \mathrm{C}(3)-\mathrm{C}(4) & 1.376(9) \\ \mathrm{C}(3)-\mathrm{H}(3) & 0.93 \\ \mathrm{C}(4)-\mathrm{C}(5) & 1.403(8) \\ \mathrm{C}(4)-\mathrm{H}(4) & 0.93 \\ \mathrm{C}(6)-\mathrm{C}(11) & 1.379(9) \\ \mathrm{C}(6)-\mathrm{C}(7) & 1.384(9) \\ \mathrm{C}(7)-\mathrm{C}(8) & 1.418(10) \\ \mathrm{C}(7)-\mathrm{H}(7) & 0.9299 \\ \mathrm{C}(8)-\mathrm{C}(9) & 1.338(12) \\ \mathrm{C}(8)-\mathrm{H}(8) & 0.93 \\ \mathrm{C}(9)-\mathrm{C}(10) & 1.374(13) \\ \mathrm{C}(9)-\mathrm{H}(9) & 0.9301 \\ \mathrm{C}(10)-\mathrm{C}(11) & 1.397(10) \\ \mathrm{C}(10)-\mathrm{H}(10) & 0.93 \\ \mathrm{C}(11)-\mathrm{H}(11) & 0.9298 \\ \mathrm{C}(12)-\mathrm{C}(13) & 1.392(8) \\ \mathrm{C}(12)-\mathrm{C}(17) & 1.399(9) \\ \mathrm{C}(13)-\mathrm{C}(14) & 1.383(9) \\ \mathrm{C}(13)-\mathrm{H}(13) & 0.9301 \\ \mathrm{C}(14)-\mathrm{C}(15) & 1.349(10) \\ \mathrm{C}(14)-\mathrm{H}(14) & 0.93 \\ \mathrm{C}(15)-\mathrm{C}(16) & 1.376(10) \\ \mathrm{C}(15)-\mathrm{H}(15) & 0.9299 \\ \mathrm{C}(16)-\mathrm{C}(17) & \\ & \\ & \\ & \end{array}$




\begin{tabular}{|c|c|}
\hline $\mathrm{C}(16)-\mathrm{H}(16)$ & 0.9298 \\
\hline $\mathrm{C}(17)-\mathrm{H}(17)$ & 0.93 \\
\hline$C(18)-C(19)$ & $1.365(8)$ \\
\hline $\mathrm{C}(18)-\mathrm{H}(18)$ & 0.9301 \\
\hline$C(19)-C(20)$ & $1.376(8)$ \\
\hline $\mathrm{C}(19)-\mathrm{H}(19)$ & 0.93 \\
\hline $\mathrm{C}(20)-\mathrm{C}(21)$ & $1.373(8)$ \\
\hline $\mathrm{C}(20)-\mathrm{H}(20)$ & 0.9299 \\
\hline $\mathrm{C}(21)-\mathrm{C}(22)$ & $1.390(8)$ \\
\hline $\mathrm{C}(21)-\mathrm{H}(21)$ & 0.9298 \\
\hline $\mathrm{C}(23)-\mathrm{C}(28)$ & $1.367(8)$ \\
\hline $\mathrm{C}(23)-\mathrm{C}(24)$ & $1.376(9)$ \\
\hline$C(24)-C(25)$ & $1.374(8)$ \\
\hline $\mathrm{C}(24)-\mathrm{H}(24)$ & 0.9301 \\
\hline$C(25)-C(26)$ & $1.386(9)$ \\
\hline $\mathrm{C}(25)-\mathrm{H}(25)$ & 0.9298 \\
\hline $\mathrm{C}(26)-\mathrm{C}(27)$ & $1.382(9)$ \\
\hline $\mathrm{C}(26)-\mathrm{H}(26)$ & 0.9298 \\
\hline $\mathrm{C}(27)-\mathrm{C}(28)$ & $1.387(8)$ \\
\hline $\mathrm{C}(27)-\mathrm{H}(27)$ & 0.9301 \\
\hline $\mathrm{C}(28)-\mathrm{H}(28)$ & 0.93 \\
\hline$C(29)-C(30)$ & $1.374(8)$ \\
\hline $\mathrm{C}(29)-\mathrm{C}(34)$ & $1.397(9)$ \\
\hline$C(30)-C(31)$ & $1.373(9)$ \\
\hline $\mathrm{C}(30)-\mathrm{H}(30)$ & 0.9301 \\
\hline $\mathrm{C}(31)-\mathrm{C}(32)$ & $1.345(10)$ \\
\hline $\mathrm{C}(31)-\mathrm{H}(31)$ & 0.93 \\
\hline $\mathrm{C}(32)-\mathrm{C}(33)$ & $1.381(10)$ \\
\hline $\mathrm{C}(32)-\mathrm{H}(32)$ & 0.93 \\
\hline$C(33)-C(34)$ & $1.379(10)$ \\
\hline $\mathrm{C}(33)-\mathrm{H}(33)$ & 0.9299 \\
\hline
\end{tabular}




\begin{tabular}{|c|c|}
\hline $\mathrm{C}(34)-\mathrm{H}(34)$ & 0.9299 \\
\hline $\mathrm{C}(35)-\mathrm{C}(36)$ & $1.359(9)$ \\
\hline $\mathrm{C}(35)-\mathrm{H}(35)$ & 0.9301 \\
\hline $\mathrm{C}(36)-\mathrm{C}(37)$ & $1.392(8)$ \\
\hline $\mathrm{C}(36)-\mathrm{H}(36)$ & 0.93 \\
\hline $\mathrm{C}(37)-\mathrm{C}(38)$ & $1.390(8)$ \\
\hline C(37)-H(37) & 0.9298 \\
\hline C(38)-C(39) & $1.389(8)$ \\
\hline C(38)-H(38) & 0.9301 \\
\hline $\mathrm{C}(40)-\mathrm{C}(45)$ & $1.384(7)$ \\
\hline $\mathrm{C}(40)-\mathrm{C}(41)$ & $1.400(8)$ \\
\hline $\mathrm{C}(41)-\mathrm{C}(42)$ & $1.392(9)$ \\
\hline $\mathrm{C}(41)-\mathrm{H}(41)$ & 0.93 \\
\hline$C(42)-C(43)$ & $1.375(9)$ \\
\hline $\mathrm{C}(42)-\mathrm{H}(42)$ & 0.9299 \\
\hline $\mathrm{C}(43)-\mathrm{C}(44)$ & $1.376(10)$ \\
\hline $\mathrm{C}(43)-\mathrm{H}(43)$ & 0.93 \\
\hline$C(44)-C(45)$ & $1.385(10)$ \\
\hline $\mathrm{C}(44)-\mathrm{H}(44)$ & 0.9301 \\
\hline $\mathrm{C}(45)-\mathrm{H}(45)$ & 0.9301 \\
\hline$C(46)-C(47)$ & $1.386(8)$ \\
\hline$C(46)-C(51)$ & $1.398(9)$ \\
\hline $\mathrm{C}(47)-\mathrm{C}(48)$ & $1.384(9)$ \\
\hline $\mathrm{C}(47)-\mathrm{H}(47)$ & 0.93 \\
\hline $\mathrm{C}(48)-\mathrm{C}(49)$ & $1.368(10)$ \\
\hline $\mathrm{C}(48)-\mathrm{H}(48)$ & 0.9298 \\
\hline$C(49)-C(50)$ & $1.364(10)$ \\
\hline C(49)-H(49) & 0.9299 \\
\hline$C(50)-C(51)$ & $1.381(8)$ \\
\hline $\mathrm{C}(50)-\mathrm{H}(50)$ & 0.93 \\
\hline $\mathrm{C}(51)-\mathrm{H}(51)$ & 0.9301 \\
\hline
\end{tabular}




\begin{tabular}{|c|c|}
\hline$C(52)-C(53)$ & $1.539(7)$ \\
\hline $\mathrm{C}(52)-\mathrm{H}(52 \mathrm{~A})$ & 0.9699 \\
\hline $\mathrm{C}(52)-\mathrm{H}(52 \mathrm{~B})$ & 0.9702 \\
\hline $\mathrm{C}(53)-\mathrm{H}(53 \mathrm{~A})$ & 0.9602 \\
\hline $\mathrm{C}(53)-\mathrm{H}(53 \mathrm{~B})$ & 0.96 \\
\hline $\mathrm{C}(53)-\mathrm{H}(53 \mathrm{C})$ & 0.9601 \\
\hline $\mathrm{C}(54)-\mathrm{C}(55)$ & $1.537(9)$ \\
\hline $\mathrm{C}(54)-\mathrm{H}(54 \mathrm{~A})$ & 0.9699 \\
\hline $\mathrm{C}(54)-\mathrm{H}(54 \mathrm{~B})$ & 0.9701 \\
\hline $\mathrm{C}(55)-\mathrm{H}(55 \mathrm{~A})$ & 0.9601 \\
\hline $\mathrm{C}(55)-\mathrm{H}(55 \mathrm{~B})$ & 0.9601 \\
\hline $\mathrm{C}(55)-\mathrm{H}(55 \mathrm{C})$ & 0.9599 \\
\hline $\mathrm{C}(56)-\mathrm{C}(57)$ & $1.334(10)$ \\
\hline $\mathrm{C}(56)-\mathrm{C}(61)$ & $1.359(10)$ \\
\hline $\mathrm{C}(56)-\mathrm{H}(56)$ & 0.9302 \\
\hline $\mathrm{C}(57)-\mathrm{C}(58)$ & $1.370(10)$ \\
\hline $\mathrm{C}(57)-\mathrm{H}(57)$ & 0.9301 \\
\hline $\mathrm{C}(58)-\mathrm{C}(59)$ & $1.364(10)$ \\
\hline $\mathrm{C}(58)-\mathrm{H}(58)$ & 0.93 \\
\hline$C(59)-C(60)$ & $1.379(9)$ \\
\hline $\mathrm{C}(59)-\mathrm{H}(59)$ & 0.9301 \\
\hline $\mathrm{C}(60)-\mathrm{C}(61)$ & $1.374(10)$ \\
\hline $\mathrm{C}(60)-\mathrm{H}(60)$ & 0.9301 \\
\hline$C(61)-C(62)$ & $1.524(10)$ \\
\hline $\mathrm{C}(62)-\mathrm{H}(62 \mathrm{~A})$ & 0.9601 \\
\hline $\mathrm{C}(62)-\mathrm{H}(62 \mathrm{~B})$ & 0.9598 \\
\hline $\mathrm{C}(62)-\mathrm{H}(62 \mathrm{C})$ & 0.96 \\
\hline $\mathrm{N}(7)-\mathrm{Zr}(1)-\mathrm{N}(2)$ & $94.24(18)$ \\
\hline $\mathrm{N}(7)-\mathrm{Zr}(1)-\mathrm{N}(4)$ & $96.47(17)$ \\
\hline $\mathrm{N}(2)-\mathrm{Zr}(1)-\mathrm{N}(4)$ & $139.44(16)$ \\
\hline
\end{tabular}




\begin{tabular}{|c|c|}
\hline $\mathrm{N}(7)-\operatorname{Zr}(1)-\mathrm{N}(6)$ & $146.52(19)$ \\
\hline $\mathrm{N}(2)-\mathrm{Zr}(1)-\mathrm{N}(6)$ & $98.58(16)$ \\
\hline $\mathrm{N}(4)-\operatorname{Zr}(1)-\mathrm{N}(6)$ & $93.62(17)$ \\
\hline $\mathrm{N}(7)-\mathrm{Zr}(1)-\mathrm{N}(5)$ & $90.69(18)$ \\
\hline $\mathrm{N}(2)-\operatorname{Zr}(1)-\mathrm{N}(5)$ & $133.40(15)$ \\
\hline $\mathrm{N}(4)-\mathrm{Zr}(1)-\mathrm{N}(5)$ & $85.52(16)$ \\
\hline $\mathrm{N}(6)-\mathrm{Zr}(1)-\mathrm{N}(5)$ & $58.34(16)$ \\
\hline $\mathrm{N}(7)-\mathrm{Zr}(1)-\mathrm{N}(1)$ & $82.90(18)$ \\
\hline $\mathrm{N}(2)-\operatorname{Zr}(1)-\mathrm{N}(1)$ & $57.71(17)$ \\
\hline $\mathrm{N}(4)-\mathrm{Zr}(1)-\mathrm{N}(1)$ & $162.60(18)$ \\
\hline $\mathrm{N}(6)-\mathrm{Zr}(1)-\mathrm{N}(1)$ & $78.21(16)$ \\
\hline $\mathrm{N}(5)-\operatorname{Zr}(1)-\mathrm{N}(1)$ & $77.11(17)$ \\
\hline $\mathrm{N}(7)-\mathrm{Zr}(1)-\mathrm{N}(3)$ & $127.42(18)$ \\
\hline $\mathrm{N}(2)-\operatorname{Zr}(1)-\mathrm{N}(3)$ & $85.30(17)$ \\
\hline $\mathrm{N}(4)-\operatorname{Zr}(1)-\mathrm{N}(3)$ & $57.45(16)$ \\
\hline $\mathrm{N}(6)-\operatorname{Zr}(1)-\mathrm{N}(3)$ & $84.56(17)$ \\
\hline $\mathrm{N}(5)-\operatorname{Zr}(1)-\mathrm{N}(3)$ & $126.33(17)$ \\
\hline $\mathrm{N}(1)-\operatorname{Zr}(1)-\mathrm{N}(3)$ & $135.47(17)$ \\
\hline $\mathrm{N}(7)-\operatorname{Zr}(1)-\mathrm{C}(39)$ & $118.79(19)$ \\
\hline $\mathrm{N}(2)-\mathrm{Zr}(1)-\mathrm{C}(39)$ & $117.81(16)$ \\
\hline $\mathrm{N}(4)-\mathrm{Zr}(1)-\mathrm{C}(39)$ & $90.47(17)$ \\
\hline $\mathrm{N}(6)-\mathrm{Zr}(1)-\mathrm{C}(39)$ & $29.11(15)$ \\
\hline $\mathrm{N}(5)-\operatorname{Zr}(1)-\mathrm{C}(39)$ & $29.26(15)$ \\
\hline $\mathrm{N}(1)-\mathrm{Zr}(1)-\mathrm{C}(39)$ & $74.87(17)$ \\
\hline $\mathrm{N}(3)-\operatorname{Zr}(1)-\mathrm{C}(39)$ & $107.16(17)$ \\
\hline $\mathrm{N}(7)-\operatorname{Zr}(1)-\mathrm{C}(5)$ & $87.49(18)$ \\
\hline $\mathrm{N}(2)-\operatorname{Zr}(1)-\mathrm{C}(5)$ & $29.00(16)$ \\
\hline $\mathrm{N}(4)-\operatorname{Zr}(1)-\mathrm{C}(5)$ & $168.38(18)$ \\
\hline $\mathrm{N}(6)-\operatorname{Zr}(1)-\mathrm{C}(5)$ & $88.96(17)$ \\
\hline $\mathrm{N}(5)-\operatorname{Zr}(1)-\mathrm{C}(5)$ & $105.41(17)$ \\
\hline $\mathrm{N}(1)-\operatorname{Zr}(1)-\mathrm{C}(5)$ & $28.73(16)$ \\
\hline
\end{tabular}




\begin{tabular}{|c|c|}
\hline $\mathrm{N}(3)-\operatorname{Zr}(1)-\mathrm{C}(5)$ & $111.64(18)$ \\
\hline$C(39)-\operatorname{Zr}(1)-C(5)$ & $97.21(17)$ \\
\hline $\mathrm{N}(7)-\mathrm{Zr}(1)-\mathrm{C}(22)$ & $116.93(17)$ \\
\hline $\mathrm{N}(2)-\mathrm{Zr}(1)-\mathrm{C}(22)$ & $113.79(17)$ \\
\hline $\mathrm{N}(4)-\operatorname{Zr}(1)-\mathrm{C}(22)$ & $28.84(15)$ \\
\hline $\mathrm{N}(6)-\mathrm{Zr}(1)-\mathrm{C}(22)$ & $85.72(17)$ \\
\hline $\mathrm{N}(5)-\operatorname{Zr}(1)-\mathrm{C}(22)$ & $104.74(17)$ \\
\hline $\mathrm{N}(1)-\operatorname{Zr}(1)-\mathrm{C}(22)$ & $159.83(15)$ \\
\hline $\mathrm{N}(3)-\operatorname{Zr}(1)-\mathrm{C}(22)$ & $28.95(16)$ \\
\hline C(39)-Zr(1)-C(22) & $96.79(17)$ \\
\hline$C(5)-\operatorname{Zr}(1)-C(22)$ & $140.56(18)$ \\
\hline $\mathrm{N}(7)-\operatorname{Zr}(1)-\mathrm{C}(52)$ & $29.70(18)$ \\
\hline $\mathrm{N}(2)-\operatorname{Zr}(1)-\mathrm{C}(52)$ & $87.17(17)$ \\
\hline $\mathrm{N}(4)-\operatorname{Zr}(1)-\mathrm{C}(52)$ & $83.28(17)$ \\
\hline $\mathrm{N}(6)-\operatorname{Zr}(1)-\mathrm{C}(52)$ & $173.86(17)$ \\
\hline $\mathrm{N}(5)-\operatorname{Zr}(1)-\mathrm{C}(52)$ & $115.99(17)$ \\
\hline$N(1)-\operatorname{Zr}(1)-C(52)$ & $103.32(17)$ \\
\hline $\mathrm{N}(3)-\operatorname{Zr}(1)-\mathrm{C}(52)$ & $98.11(18)$ \\
\hline$C(39)-\operatorname{Zr}(1)-C(52)$ & $145.24(17)$ \\
\hline$C(5)-\operatorname{Zr}(1)-C(52)$ & $95.13(17)$ \\
\hline $\mathrm{C}(22)-\mathrm{Zr}(1)-\mathrm{C}(52)$ & $93.95(17)$ \\
\hline $\mathrm{N}(2)-\mathrm{P}(1)-\mathrm{C}(12)$ & $106.4(3)$ \\
\hline $\mathrm{N}(2)-\mathrm{P}(1)-\mathrm{C}(6)$ & $103.9(3)$ \\
\hline $\mathrm{C}(12)-\mathrm{P}(1)-\mathrm{C}(6)$ & $102.4(3)$ \\
\hline $\mathrm{N}(4)-\mathrm{P}(2)-\mathrm{C}(23)$ & $106.0(3)$ \\
\hline $\mathrm{N}(4)-\mathrm{P}(2)-\mathrm{C}(29)$ & $106.1(2)$ \\
\hline $\mathrm{C}(23)-\mathrm{P}(2)-\mathrm{C}(29)$ & $102.3(3)$ \\
\hline $\mathrm{N}(6)-\mathrm{P}(3)-\mathrm{C}(40)$ & $105.6(3)$ \\
\hline $\mathrm{N}(6)-\mathrm{P}(3)-\mathrm{C}(46)$ & $106.1(2)$ \\
\hline $\mathrm{C}(40)-\mathrm{P}(3)-\mathrm{C}(46)$ & $102.3(3)$ \\
\hline $\mathrm{C}(5)-\mathrm{N}(1)-\mathrm{C}(1)$ & $120.1(6)$ \\
\hline
\end{tabular}




\begin{tabular}{|c|c|}
\hline $\mathrm{C}(5)-\mathrm{N}(1)-\operatorname{Zr}(1)$ & $94.3(4)$ \\
\hline $\mathrm{C}(1)-\mathrm{N}(1)-\operatorname{Zr}(1)$ & $145.5(5)$ \\
\hline $\mathrm{C}(5)-\mathrm{N}(2)-\mathrm{P}(1)$ & $127.1(4)$ \\
\hline $\mathrm{C}(5)-\mathrm{N}(2)-\mathrm{Zr}(1)$ & $98.3(4)$ \\
\hline $\mathrm{P}(1)-\mathrm{N}(2)-\mathrm{Zr}(1)$ & $134.0(2)$ \\
\hline $\mathrm{C}(18)-\mathrm{N}(3)-\mathrm{C}(22)$ & $119.5(5)$ \\
\hline $\mathrm{C}(18)-\mathrm{N}(3)-\mathrm{Zr}(1)$ & $144.8(4)$ \\
\hline $\mathrm{C}(22)-\mathrm{N}(3)-\mathrm{Zr}(1)$ & $94.1(3)$ \\
\hline $\mathrm{C}(22)-\mathrm{N}(4)-\mathrm{P}(2)$ & $128.0(4)$ \\
\hline $\mathrm{C}(22)-\mathrm{N}(4)-\mathrm{Zr}(1)$ & $98.3(3)$ \\
\hline $\mathrm{P}(2)-\mathrm{N}(4)-\mathrm{Zr}(1)$ & $133.5(2)$ \\
\hline $\mathrm{C}(35)-\mathrm{N}(5)-\mathrm{C}(39)$ & $120.7(5)$ \\
\hline $\mathrm{C}(35)-\mathrm{N}(5)-\mathrm{Zr}(1)$ & $144.9(4)$ \\
\hline $\mathrm{C}(39)-\mathrm{N}(5)-\mathrm{Zr}(1)$ & $94.3(4)$ \\
\hline $\mathrm{C}(39)-\mathrm{N}(6)-\mathrm{P}(3)$ & $128.4(4)$ \\
\hline $\mathrm{C}(39)-\mathrm{N}(6)-\mathrm{Zr}(1)$ & $97.5(3)$ \\
\hline $\mathrm{P}(3)-\mathrm{N}(6)-\mathrm{Zr}(1)$ & $132.3(3)$ \\
\hline $\mathrm{C}(52)-\mathrm{N}(7)-\mathrm{C}(54)$ & $116.6(5)$ \\
\hline $\mathrm{C}(52)-\mathrm{N}(7)-\mathrm{Zr}(1)$ & $106.5(3)$ \\
\hline $\mathrm{C}(54)-\mathrm{N}(7)-\mathrm{Zr}(1)$ & $136.8(4)$ \\
\hline $\mathrm{N}(1)-\mathrm{C}(1)-\mathrm{C}(2)$ & $122.3(7)$ \\
\hline $\mathrm{N}(1)-\mathrm{C}(1)-\mathrm{H}(1)$ & 118.6 \\
\hline $\mathrm{C}(2)-\mathrm{C}(1)-\mathrm{H}(1)$ & 119.1 \\
\hline $\mathrm{C}(1)-\mathrm{C}(2)-\mathrm{C}(3)$ & $118.2(7)$ \\
\hline $\mathrm{C}(1)-\mathrm{C}(2)-\mathrm{H}(2)$ & 120.5 \\
\hline $\mathrm{C}(3)-\mathrm{C}(2)-\mathrm{H}(2)$ & 121.3 \\
\hline$C(2)-C(3)-C(4)$ & $120.9(7)$ \\
\hline $\mathrm{C}(2)-\mathrm{C}(3)-\mathrm{H}(3)$ & 119.5 \\
\hline $\mathrm{C}(4)-\mathrm{C}(3)-\mathrm{H}(3)$ & 119.5 \\
\hline$C(3)-C(4)-C(5)$ & $118.5(7)$ \\
\hline $\mathrm{C}(3)-\mathrm{C}(4)-\mathrm{H}(4)$ & 120.7 \\
\hline
\end{tabular}




$\begin{array}{ll}\mathrm{C}(5)-\mathrm{C}(4)-\mathrm{H}(4) & 120.8 \\ \mathrm{~N}(1)-\mathrm{C}(5)-\mathrm{N}(2) & 109.6(5) \\ \mathrm{N}(1)-\mathrm{C}(5)-\mathrm{C}(4) & 119.9(6) \\ \mathrm{N}(2)-\mathrm{C}(5)-\mathrm{C}(4) & 130.5(6) \\ \mathrm{N}(1)-\mathrm{C}(5)-\mathrm{Zr}(1) & 56.9(3) \\ \mathrm{N}(2)-\mathrm{C}(5)-\mathrm{Zr}(1) & 52.7(3) \\ \mathrm{C}(4)-\mathrm{C}(5)-\mathrm{Zr}(1) & 176.5(5) \\ \mathrm{C}(11)-\mathrm{C}(6)-\mathrm{C}(7) & 118.2(6) \\ \mathrm{C}(11)-\mathrm{C}(6)-\mathrm{P}(1) & 126.0(6) \\ \mathrm{C}(7)-\mathrm{C}(6)-\mathrm{P}(1) & 115.8(6) \\ \mathrm{C}(6)-\mathrm{C}(7)-\mathrm{C}(8) & 120.1(8) \\ \mathrm{C}(6)-\mathrm{C}(7)-\mathrm{H}(7) & 120.3 \\ \mathrm{C}(8)-\mathrm{C}(7)-\mathrm{H}(7) & 119.5 \\ \mathrm{C}(9)-\mathrm{C}(8)-\mathrm{C}(7) & 120.2(9) \\ \mathrm{C}(9)-\mathrm{C}(8)-\mathrm{H}(8) & 120 \\ \mathrm{C}(7)-\mathrm{C}(8)-\mathrm{H}(8) & 119.8 \\ \mathrm{C}(8)-\mathrm{C}(9)-\mathrm{C}(10) & 120.7(9) \\ \mathrm{C}(8)-\mathrm{C}(9)-\mathrm{H}(9) & 119.8 \\ \mathrm{C}(10)-\mathrm{C}(9)-\mathrm{H}(9) & 119.4 \\ \mathrm{C}(9)-\mathrm{C}(10)-\mathrm{C}(11) & 119.5(10) \\ \mathrm{C}(9)-\mathrm{C}(10)-\mathrm{H}(10) & 120.8 \\ \mathrm{C}(11)-\mathrm{C}(10)-\mathrm{H}(10) & 119.7 \\ \mathrm{C}(6)-\mathrm{C}(11)-\mathrm{C}(10) & 121.2(9) \\ \mathrm{C}(6)-\mathrm{C}(11)-\mathrm{H}(11) & 119.6 \\ \mathrm{C}(10)-\mathrm{C}(11)-\mathrm{H}(11) & 119.3 \\ \mathrm{C}(13)-\mathrm{C}(12)-\mathrm{C}(17) & 118.6(6) \\ \mathrm{C}(13)-\mathrm{C}(12)-\mathrm{P}(1) & 121.8(5) \\ \mathrm{C}(17)-\mathrm{C}(12)-\mathrm{P}(1) & 119.3(5) \\ \mathrm{C}(14)-\mathrm{C}(13)-\mathrm{C}(12) & 119.1(7) \\ \mathrm{C}(14)-\mathrm{C}(13)-\mathrm{H}(13) & 120.5 \\ \mathrm{C}(12)-\mathrm{C}(13)-\mathrm{H}(13) & \\ & \\ & 120.4 \\ & \end{array}$




\begin{tabular}{|c|c|}
\hline$C(15)-C(14)-C(13)$ & $122.7(7)$ \\
\hline $\mathrm{C}(15)-\mathrm{C}(14)-\mathrm{H}(14)$ & 118.7 \\
\hline $\mathrm{C}(13)-\mathrm{C}(14)-\mathrm{H}(14)$ & 118.6 \\
\hline$C(14)-C(15)-C(16)$ & $119.4(7)$ \\
\hline $\mathrm{C}(14)-\mathrm{C}(15)-\mathrm{H}(15)$ & 120.3 \\
\hline $\mathrm{C}(16)-\mathrm{C}(15)-\mathrm{H}(15)$ & 120.3 \\
\hline$C(15)-C(16)-C(17)$ & $119.8(7)$ \\
\hline $\mathrm{C}(15)-\mathrm{C}(16)-\mathrm{H}(16)$ & 120 \\
\hline$C(17)-C(16)-H(16)$ & 120.2 \\
\hline$C(16)-C(17)-C(12)$ & $120.3(7)$ \\
\hline$C(16)-C(17)-H(17)$ & 119.5 \\
\hline $\mathrm{C}(12)-\mathrm{C}(17)-\mathrm{H}(17)$ & 120.2 \\
\hline $\mathrm{N}(3)-\mathrm{C}(18)-\mathrm{C}(19)$ & $123.0(6)$ \\
\hline $\mathrm{N}(3)-\mathrm{C}(18)-\mathrm{H}(18)$ & 118.6 \\
\hline $\mathrm{C}(19)-\mathrm{C}(18)-\mathrm{H}(18)$ & 118.4 \\
\hline$C(18)-C(19)-C(20)$ & $118.0(6)$ \\
\hline $\mathrm{C}(18)-\mathrm{C}(19)-\mathrm{H}(19)$ & 121.1 \\
\hline $\mathrm{C}(20)-\mathrm{C}(19)-\mathrm{H}(19)$ & 120.9 \\
\hline$C(21)-C(20)-C(19)$ & $120.0(6)$ \\
\hline $\mathrm{C}(21)-\mathrm{C}(20)-\mathrm{H}(20)$ & 120 \\
\hline $\mathrm{C}(19)-\mathrm{C}(20)-\mathrm{H}(20)$ & 120 \\
\hline$C(20)-C(21)-C(22)$ & $119.8(6)$ \\
\hline $\mathrm{C}(20)-\mathrm{C}(21)-\mathrm{H}(21)$ & 120 \\
\hline $\mathrm{C}(22)-\mathrm{C}(21)-\mathrm{H}(21)$ & 120.2 \\
\hline $\mathrm{N}(3)-\mathrm{C}(22)-\mathrm{N}(4)$ & $108.9(5)$ \\
\hline $\mathrm{N}(3)-\mathrm{C}(22)-\mathrm{C}(21)$ & $119.7(5)$ \\
\hline $\mathrm{N}(4)-\mathrm{C}(22)-\mathrm{C}(21)$ & $131.4(6)$ \\
\hline $\mathrm{N}(3)-\mathrm{C}(22)-\operatorname{Zr}(1)$ & $57.0(3)$ \\
\hline $\mathrm{N}(4)-\mathrm{C}(22)-\mathrm{Zr}(1)$ & $52.8(3)$ \\
\hline$C(21)-C(22)-\operatorname{Zr}(1)$ & $169.3(4)$ \\
\hline $\mathrm{C}(28)-\mathrm{C}(23)-\mathrm{C}(24)$ & $117.1(6)$ \\
\hline
\end{tabular}




\begin{tabular}{|c|c|}
\hline $\mathrm{C}(28)-\mathrm{C}(23)-\mathrm{P}(2)$ & $120.1(5)$ \\
\hline $\mathrm{C}(24)-\mathrm{C}(23)-\mathrm{P}(2)$ & $122.2(5)$ \\
\hline$C(25)-C(24)-C(23)$ & $122.2(6)$ \\
\hline $\mathrm{C}(25)-\mathrm{C}(24)-\mathrm{H}(24)$ & 119 \\
\hline $\mathrm{C}(23)-\mathrm{C}(24)-\mathrm{H}(24)$ & 118.8 \\
\hline$C(24)-C(25)-C(26)$ & $120.1(7)$ \\
\hline $\mathrm{C}(24)-\mathrm{C}(25)-\mathrm{H}(25)$ & 119.9 \\
\hline $\mathrm{C}(26)-\mathrm{C}(25)-\mathrm{H}(25)$ & 120 \\
\hline$C(27)-C(26)-C(25)$ & $118.5(6)$ \\
\hline $\mathrm{C}(27)-\mathrm{C}(26)-\mathrm{H}(26)$ & 120.7 \\
\hline $\mathrm{C}(25)-\mathrm{C}(26)-\mathrm{H}(26)$ & 120.8 \\
\hline$C(26)-C(27)-C(28)$ & $119.8(6)$ \\
\hline $\mathrm{C}(26)-\mathrm{C}(27)-\mathrm{H}(27)$ & 120.1 \\
\hline $\mathrm{C}(28)-\mathrm{C}(27)-\mathrm{H}(27)$ & 120.1 \\
\hline$C(23)-C(28)-C(27)$ & $122.2(7)$ \\
\hline $\mathrm{C}(23)-\mathrm{C}(28)-\mathrm{H}(28)$ & 119 \\
\hline $\mathrm{C}(27)-\mathrm{C}(28)-\mathrm{H}(28)$ & 118.8 \\
\hline$C(30)-C(29)-C(34)$ & $115.8(7)$ \\
\hline $\mathrm{C}(30)-\mathrm{C}(29)-\mathrm{P}(2)$ & $128.5(6)$ \\
\hline $\mathrm{C}(34)-\mathrm{C}(29)-\mathrm{P}(2)$ & $115.5(6)$ \\
\hline $\mathrm{C}(31)-\mathrm{C}(30)-\mathrm{C}(29)$ & $123.1(7)$ \\
\hline $\mathrm{C}(31)-\mathrm{C}(30)-\mathrm{H}(30)$ & 118 \\
\hline $\mathrm{C}(29)-\mathrm{C}(30)-\mathrm{H}(30)$ & 118.8 \\
\hline$C(32)-C(31)-C(30)$ & $119.7(8)$ \\
\hline $\mathrm{C}(32)-\mathrm{C}(31)-\mathrm{H}(31)$ & 119.8 \\
\hline $\mathrm{C}(30)-\mathrm{C}(31)-\mathrm{H}(31)$ & 120.5 \\
\hline $\mathrm{C}(31)-\mathrm{C}(32)-\mathrm{C}(33)$ & $120.3(9)$ \\
\hline $\mathrm{C}(31)-\mathrm{C}(32)-\mathrm{H}(32)$ & 119.7 \\
\hline $\mathrm{C}(33)-\mathrm{C}(32)-\mathrm{H}(32)$ & 119.9 \\
\hline$C(34)-C(33)-C(32)$ & $119.2(8)$ \\
\hline $\mathrm{C}(34)-\mathrm{C}(33)-\mathrm{H}(33)$ & 120.4 \\
\hline
\end{tabular}




$\begin{array}{ll}\mathrm{C}(32)-\mathrm{C}(33)-\mathrm{H}(33) & 120.3 \\ \mathrm{C}(33)-\mathrm{C}(34)-\mathrm{C}(29) & 121.8(7) \\ \mathrm{C}(33)-\mathrm{C}(34)-\mathrm{H}(34) & 119 \\ \mathrm{C}(29)-\mathrm{C}(34)-\mathrm{H}(34) & 119.1 \\ \mathrm{~N}(5)-\mathrm{C}(35)-\mathrm{C}(36) & 121.3(6) \\ \mathrm{N}(5)-\mathrm{C}(35)-\mathrm{H}(35) & 119.5 \\ \mathrm{C}(36)-\mathrm{C}(35)-\mathrm{H}(35) & 119.2 \\ \mathrm{C}(35)-\mathrm{C}(36)-\mathrm{C}(37) & 120.2(6) \\ \mathrm{C}(35)-\mathrm{C}(36)-\mathrm{H}(36) & 120.2 \\ \mathrm{C}(37)-\mathrm{C}(36)-\mathrm{H}(36) & 119.7 \\ \mathrm{C}(36)-\mathrm{C}(37)-\mathrm{C}(38) & 118.4(6) \\ \mathrm{C}(36)-\mathrm{C}(37)-\mathrm{H}(37) & 120.8 \\ \mathrm{C}(38)-\mathrm{C}(37)-\mathrm{H}(37) & 120.7 \\ \mathrm{C}(39)-\mathrm{C}(38)-\mathrm{C}(37) & 119.6(6) \\ \mathrm{C}(39)-\mathrm{C}(38)-\mathrm{H}(38) & 120.3 \\ \mathrm{C}(37)-\mathrm{C}(38)-\mathrm{H}(38) & 120.1 \\ \mathrm{~N}(5)-\mathrm{C}(39)-\mathrm{N}(6) & 109.7(5) \\ \mathrm{N}(5)-\mathrm{C}(39)-\mathrm{C}(38) & 119.8(6) \\ \mathrm{N}(6)-\mathrm{C}(39)-\mathrm{C}(38) & 130.5(5) \\ \mathrm{N}(5)-\mathrm{C}(39)-\mathrm{Zr}(1) & 56.4(3) \\ \mathrm{N}(6)-\mathrm{C}(39)-\mathrm{Zr}(1) & 53.4(3) \\ \mathrm{C}(38)-\mathrm{C}(39)-\mathrm{Zr}(1) & 175.4(5) \\ \mathrm{C}(45)-\mathrm{C}(40)-\mathrm{C}(41) & 118.0(6) \\ \mathrm{C}(45)-\mathrm{C}(40)-\mathrm{P}(3) & 119.8(5) \\ \mathrm{C}(41)-\mathrm{C}(40)-\mathrm{P}(3) & 121.2(5) \\ \mathrm{C}(42)-\mathrm{C}(41)-\mathrm{C}(40) & 121.3(6) \\ \mathrm{C}(42)-\mathrm{C}(41)-\mathrm{H}(41) & 119.5 \\ \mathrm{C}(40)-\mathrm{C}(41)-\mathrm{H}(41) & 119.2 \\ \mathrm{C}(43)-\mathrm{C}(42)-\mathrm{C}(41) & 120.4(7) \\ \mathrm{C}(43)-\mathrm{C}(42)-\mathrm{H}(42) & \mathrm{C}(42)-\mathrm{H}(42) \\ & 119.8 \\ & \end{array}$




\begin{tabular}{|c|c|}
\hline$C(44)-C(43)-C(42)$ & $117.8(7)$ \\
\hline $\mathrm{C}(44)-\mathrm{C}(43)-\mathrm{H}(43)$ & 121.3 \\
\hline $\mathrm{C}(42)-\mathrm{C}(43)-\mathrm{H}(43)$ & 120.8 \\
\hline$C(43)-C(44)-C(45)$ & $123.0(7)$ \\
\hline $\mathrm{C}(43)-\mathrm{C}(44)-\mathrm{H}(44)$ & 118.4 \\
\hline $\mathrm{C}(45)-\mathrm{C}(44)-\mathrm{H}(44)$ & 118.6 \\
\hline$C(44)-C(45)-C(40)$ & $119.5(7)$ \\
\hline $\mathrm{C}(44)-\mathrm{C}(45)-\mathrm{H}(45)$ & 120.3 \\
\hline $\mathrm{C}(40)-\mathrm{C}(45)-\mathrm{H}(45)$ & 120.3 \\
\hline$C(47)-C(46)-C(51)$ & $116.3(6)$ \\
\hline $\mathrm{C}(47)-\mathrm{C}(46)-\mathrm{P}(3)$ & $128.6(6)$ \\
\hline $\mathrm{C}(51)-\mathrm{C}(46)-\mathrm{P}(3)$ & $115.1(5)$ \\
\hline$C(48)-C(47)-C(46)$ & $121.7(7)$ \\
\hline $\mathrm{C}(48)-\mathrm{C}(47)-\mathrm{H}(47)$ & 119.4 \\
\hline $\mathrm{C}(46)-\mathrm{C}(47)-\mathrm{H}(47)$ & 118.9 \\
\hline$C(49)-C(48)-C(47)$ & $120.2(8)$ \\
\hline $\mathrm{C}(49)-\mathrm{C}(48)-\mathrm{H}(48)$ & 120.1 \\
\hline $\mathrm{C}(47)-\mathrm{C}(48)-\mathrm{H}(48)$ & 119.7 \\
\hline $\mathrm{C}(50)-\mathrm{C}(49)-\mathrm{C}(48)$ & $120.0(7)$ \\
\hline $\mathrm{C}(50)-\mathrm{C}(49)-\mathrm{H}(49)$ & 120.1 \\
\hline $\mathrm{C}(48)-\mathrm{C}(49)-\mathrm{H}(49)$ & 119.9 \\
\hline$C(49)-C(50)-C(51)$ & $119.7(7)$ \\
\hline $\mathrm{C}(49)-\mathrm{C}(50)-\mathrm{H}(50)$ & 120 \\
\hline $\mathrm{C}(51)-\mathrm{C}(50)-\mathrm{H}(50)$ & 120.3 \\
\hline $\mathrm{C}(50)-\mathrm{C}(51)-\mathrm{C}(46)$ & $122.1(7)$ \\
\hline $\mathrm{C}(50)-\mathrm{C}(51)-\mathrm{H}(51)$ & 119 \\
\hline $\mathrm{C}(46)-\mathrm{C}(51)-\mathrm{H}(51)$ & 118.9 \\
\hline $\mathrm{N}(7)-\mathrm{C}(52)-\mathrm{C}(53)$ & $114.2(5)$ \\
\hline $\mathrm{N}(7)-\mathrm{C}(52)-\operatorname{Zr}(1)$ & $43.8(2)$ \\
\hline$C(53)-C(52)-Z r(1)$ & $129.3(4)$ \\
\hline $\mathrm{N}(7)-\mathrm{C}(52)-\mathrm{H}(52 \mathrm{~A})$ & 108.5 \\
\hline
\end{tabular}




$\begin{array}{ll}\mathrm{C}(53)-\mathrm{C}(52)-\mathrm{H}(52 \mathrm{~A}) & 108.7 \\ \mathrm{Zr}(1)-\mathrm{C}(52)-\mathrm{H}(52 \mathrm{~A}) & 121.3 \\ \mathrm{~N}(7)-\mathrm{C}(52)-\mathrm{H}(52 \mathrm{~B}) & 108.7 \\ \mathrm{C}(53)-\mathrm{C}(52)-\mathrm{H}(52 \mathrm{~B}) & 108.9 \\ \mathrm{Zr}(1)-\mathrm{C}(52)-\mathrm{H}(52 \mathrm{~B}) & 64.9 \\ \mathrm{H}(52 \mathrm{~A})-\mathrm{C}(52)-\mathrm{H}(52 \mathrm{~B}) & 107.6 \\ \mathrm{C}(52)-\mathrm{C}(53)-\mathrm{H}(53 \mathrm{~A}) & 109.1 \\ \mathrm{C}(52)-\mathrm{C}(53)-\mathrm{H}(53 \mathrm{~B}) & 109.5 \\ \mathrm{H}(53 \mathrm{~A})-\mathrm{C}(53)-\mathrm{H}(53 \mathrm{~B}) & 109.5 \\ \mathrm{C}(52)-\mathrm{C}(53)-\mathrm{H}(53 \mathrm{C}) & 109.8 \\ \mathrm{H}(53 \mathrm{~A})-\mathrm{C}(53)-\mathrm{H}(53 \mathrm{C}) & 109.5 \\ \mathrm{H}(53 \mathrm{~B})-\mathrm{C}(53)-\mathrm{H}(53 \mathrm{C}) & 109.5 \\ \mathrm{~N}(7)-\mathrm{C}(54)-\mathrm{C}(55) & 114.7(6) \\ \mathrm{N}(7)-\mathrm{C}(54)-\mathrm{H}(54 \mathrm{~A}) & 108.8 \\ \mathrm{C}(55)-\mathrm{C}(54)-\mathrm{H}(54 \mathrm{~A}) & 108.9 \\ \mathrm{~N}(7)-\mathrm{C}(54)-\mathrm{H}(54 \mathrm{~B}) & 108.6 \\ \mathrm{C}(55)-\mathrm{C}(54)-\mathrm{H}(54 \mathrm{~B}) & 108.1 \\ \mathrm{H}(54 \mathrm{~A})-\mathrm{C}(54)-\mathrm{H}(54 \mathrm{~B}) & 107.5 \\ \mathrm{C}(54)-\mathrm{C}(55)-\mathrm{H}(55 \mathrm{~A}) & 109.5 \\ \mathrm{C}(54)-\mathrm{C}(55)-\mathrm{H}(55 \mathrm{~B}) & 109.9 \\ \mathrm{H}(55 \mathrm{~A})-\mathrm{C}(55)-\mathrm{H}(55 \mathrm{~B}) & 109.5 \\ \mathrm{C}(54)-\mathrm{C}(55)-\mathrm{H}(55 \mathrm{C}) & 109.1 \\ \mathrm{H}(55 \mathrm{~A})-\mathrm{C}(55)-\mathrm{H}(55 \mathrm{C}) & 109.5 \\ \mathrm{H}(55 \mathrm{~B})-\mathrm{C}(55)-\mathrm{H}(55 \mathrm{C}) & 109.5 \\ \mathrm{C}(57)-\mathrm{C}(56)-\mathrm{C}(61) & 120.6(8) \\ \mathrm{C}(57)-\mathrm{C}(56)-\mathrm{H}(56) & 119.6 \\ \mathrm{C}(61)-\mathrm{C}(56)-\mathrm{H}(56) & 119.9 \\ \mathrm{C}(56)-\mathrm{C}(57)-\mathrm{C}(58) & 121.6(8) \\ \mathrm{C}(56)-\mathrm{C}(57)-\mathrm{H}(57) & \\ \mathrm{C}(58)-\mathrm{C}(57)-\mathrm{H}(57) & \\ \mathrm{C}(59)-\mathrm{C}(58)-\mathrm{C}(57) & 19.3 \\ & \end{array}$




$\begin{array}{ll}\mathrm{C}(59)-\mathrm{C}(58)-\mathrm{H}(58) & 120.4 \\ \mathrm{C}(57)-\mathrm{C}(58)-\mathrm{H}(58) & 120.8 \\ \mathrm{C}(58)-\mathrm{C}(59)-\mathrm{C}(60) & 119.9(8) \\ \mathrm{C}(58)-\mathrm{C}(59)-\mathrm{H}(59) & 120.4 \\ \mathrm{C}(60)-\mathrm{C}(59)-\mathrm{H}(59) & 119.8 \\ \mathrm{C}(61)-\mathrm{C}(60)-\mathrm{C}(59) & 119.9(8) \\ \mathrm{C}(61)-\mathrm{C}(60)-\mathrm{H}(60) & 120.3 \\ \mathrm{C}(59)-\mathrm{C}(60)-\mathrm{H}(60) & 119.9 \\ \mathrm{C}(56)-\mathrm{C}(61)-\mathrm{C}(60) & 119.3(8) \\ \mathrm{C}(56)-\mathrm{C}(61)-\mathrm{C}(62) & 119.6(8) \\ \mathrm{C}(60)-\mathrm{C}(61)-\mathrm{C}(62) & 121.1(8) \\ \mathrm{C}(61)-\mathrm{C}(62)-\mathrm{H}(62 \mathrm{~A}) & 109.4 \\ \mathrm{C}(61)-\mathrm{C}(62)-\mathrm{H}(62 \mathrm{~B}) & 109.5 \\ \mathrm{H}(62 \mathrm{~A})-\mathrm{C}(62)-\mathrm{H}(62 \mathrm{~B}) & 109.5 \\ \mathrm{C}(61)-\mathrm{C}(62)-\mathrm{H}(62 \mathrm{C}) & 109.6 \\ \mathrm{H}(62 \mathrm{~A})-\mathrm{C}(62)-\mathrm{H}(62 \mathrm{C}) & 109.4 \\ \mathrm{H}(62 \mathrm{~B})-\mathrm{C}(62)-\mathrm{H}(62 \mathrm{C}) & 109.5\end{array}$

Table 4. Anisotropic displacement parameters $\left(\mathrm{A}^{2} \times 10^{3}\right)$ for complex 13.

The anisotropic displacement factor exponent takes the form:

$-2 \pi^{2}\left[h^{2} a^{* \wedge} 2 \mathrm{U} 11+\ldots+2 \mathrm{~h} \mathrm{k} \mathrm{a} \mathrm{b}^{*} \mathrm{U} 12\right]$

\begin{tabular}{lllllll}
\hline & U11 & U22 & U33 & U23 & U13 & U12 \\
\hline $\operatorname{Zr}(1)$ & $34(1)$ & $32(1)$ & $37(1)$ & $0(1)$ & $2(1)$ & $0(1)$ \\
$\mathrm{P}(1)$ & $42(1)$ & $56(1)$ & $46(1)$ & $11(1)$ & $2(1)$ & $-3(1)$ \\
$\mathrm{P}(2)$ & $39(1)$ & $38(1)$ & $44(1)$ & $4(1)$ & $0(1)$ & $0(1)$ \\
$\mathrm{P}(3)$ & $44(1)$ & $37(1)$ & $44(1)$ & $-2(1)$ & $5(1)$ & $-3(1)$ \\
$\mathrm{N}(1)$ & $44(4)$ & $41(3)$ & $38(4)$ & $-1(3)$ & $3(3)$ & $3(2)$ \\
$\mathrm{N}(2)$ & $33(3)$ & $38(3)$ & $38(4)$ & $1(2)$ & $3(2)$ & $1(2)$ \\
$\mathrm{N}(3)$ & $36(3)$ & $37(3)$ & $45(4)$ & $8(3)$ & $2(3)$ & $-4(2)$ \\
$\mathrm{N}(4)$ & $32(3)$ & $31(2)$ & $45(4)$ & $4(2)$ & $7(3)$ & $-7(2)$
\end{tabular}




\begin{tabular}{|c|c|c|c|c|c|c|}
\hline$N(5)$ & $34(3)$ & $35(3)$ & $32(3)$ & $4(2)$ & $4(2)$ & $1(2)$ \\
\hline $\mathrm{N}(6)$ & $40(3)$ & $32(2)$ & $45(4)$ & $5(2)$ & $7(3)$ & $-3(2)$ \\
\hline $\mathrm{N}(7)$ & $42(3)$ & $32(3)$ & $51(4)$ & $-4(3)$ & $6(3)$ & $-9(2)$ \\
\hline $\mathrm{C}(1)$ & $47(4)$ & $53(4)$ & $55(5)$ & $-11(4)$ & $3(4)$ & $-8(3)$ \\
\hline$C(2)$ & $37(4)$ & $70(5)$ & $68(7)$ & $-19(5)$ & $-1(4)$ & $15(4)$ \\
\hline$C(3)$ & $46(5)$ & $70(5)$ & $62(6)$ & $-8(4)$ & $-9(4)$ & $17(4)$ \\
\hline$C(4)$ & $55(5)$ & $48(4)$ & $47(5)$ & $3(3)$ & $-4(4)$ & $4(3)$ \\
\hline$C(5)$ & $47(4)$ & $29(3)$ & $42(5)$ & $0(3)$ & $-3(4)$ & $0(3)$ \\
\hline$C(6)$ & $48(5)$ & $55(4)$ & $62(6)$ & $18(4)$ & $-2(4)$ & $-17(3)$ \\
\hline$C(7)$ & $81(6)$ & $53(4)$ & $72(7)$ & $7(4)$ & $-9(5)$ & $-18(4)$ \\
\hline $\mathrm{C}(8)$ & $131(8)$ & $61(6)$ & $109(10)$ & $6(6)$ & $-16(7)$ & $-26(5)$ \\
\hline $\mathrm{C}(9)$ & 199(12) & $48(5)$ & $151(13)$ & $18(7)$ & $-33(11)$ & $-24(7)$ \\
\hline$C(10)$ & $140(9)$ & $79(6)$ & $119(11)$ & $55(7)$ & $-28(8)$ & $-9(6)$ \\
\hline$C(11)$ & $100(6)$ & $62(5)$ & $78(7)$ & $20(5)$ & $-13(6)$ & $-8(5)$ \\
\hline$C(12)$ & $49(4)$ & $47(4)$ & $33(5)$ & $7(3)$ & $-1(3)$ & $5(4)$ \\
\hline$C(13)$ & $64(5)$ & $39(3)$ & $39(5)$ & $0(3)$ & $1(3)$ & $5(4)$ \\
\hline$C(14)$ & $74(5)$ & $53(4)$ & $58(6)$ & $-8(4)$ & $0(5)$ & $-11(4)$ \\
\hline$C(15)$ & $90(7)$ & $70(5)$ & $51(6)$ & $-16(4)$ & $-9(5)$ & $-3(4)$ \\
\hline$C(16)$ & $75(6)$ & 111(6) & $40(6)$ & $1(5)$ & $6(5)$ & $9(5)$ \\
\hline$C(17)$ & $56(5)$ & $91(5)$ & $53(7)$ & $14(5)$ & $4(4)$ & $-1(4)$ \\
\hline $\mathrm{C}(18)$ & $50(4)$ & $39(4)$ & $59(6)$ & $8(3)$ & $-4(4)$ & $-1(3)$ \\
\hline$C(19)$ & $42(4)$ & $44(4)$ & $70(6)$ & $9(4)$ & $-2(4)$ & $-10(3)$ \\
\hline$C(20)$ & $34(4)$ & $50(4)$ & $72(6)$ & $6(4)$ & $-6(4)$ & $-14(3)$ \\
\hline $\mathrm{C}(21)$ & $43(4)$ & $48(4)$ & $44(5)$ & $-2(3)$ & $-6(3)$ & $5(3)$ \\
\hline$C(22)$ & $39(4)$ & $34(3)$ & $37(4)$ & $-3(3)$ & 1(3) & $8(3)$ \\
\hline$C(23)$ & $31(4)$ & $33(3)$ & $45(5)$ & $2(3)$ & $-2(3)$ & $-4(3)$ \\
\hline$C(24)$ & $43(4)$ & $38(3)$ & $66(6)$ & $0(4)$ & $-4(4)$ & $8(3)$ \\
\hline$C(25)$ & $64(5)$ & $49(4)$ & $56(6)$ & $-11(4)$ & $7(4)$ & $0(4)$ \\
\hline$C(26)$ & $51(5)$ & $43(4)$ & $81(7)$ & $-16(4)$ & $12(4)$ & $2(3)$ \\
\hline $\mathrm{C}(27)$ & $47(4)$ & $45(4)$ & $82(7)$ & $2(4)$ & $-15(4)$ & $4(4)$ \\
\hline $\mathrm{C}(28)$ & $35(4)$ & $41(4)$ & $65(6)$ & $4(4)$ & $-7(4)$ & $-2(3)$ \\
\hline
\end{tabular}




\begin{tabular}{|c|c|c|c|c|c|c|}
\hline$C(29)$ & $53(4)$ & $42(4)$ & $35(5)$ & $4(3)$ & $-4(4)$ & $-5(3)$ \\
\hline$C(30)$ & $49(5)$ & $53(4)$ & $50(6)$ & $-1(4)$ & $-4(4)$ & $-16(3)$ \\
\hline $\mathrm{C}(31)$ & $70(6)$ & $61(5)$ & $66(7)$ & $-2(5)$ & $-12(5)$ & $-18(4)$ \\
\hline$C(32)$ & $134(9)$ & $65(5)$ & $51(7)$ & $-10(5)$ & $-20(6)$ & $-22(5)$ \\
\hline$C(33)$ & $108(7)$ & $89(6)$ & $44(7)$ & $-23(5)$ & $-2(5)$ & $16(5)$ \\
\hline$C(34)$ & $66(5)$ & $58(4)$ & $66(7)$ & $-5(4)$ & $-7(5)$ & $12(4)$ \\
\hline$C(35)$ & $45(4)$ & $34(3)$ & $60(6)$ & $-1(3)$ & $7(4)$ & $-5(3)$ \\
\hline$C(36)$ & $50(4)$ & $55(4)$ & $57(6)$ & $15(4)$ & $11(4)$ & $-7(3)$ \\
\hline$C(37)$ & $52(4)$ & $53(4)$ & $44(5)$ & $2(4)$ & $12(4)$ & $8(3)$ \\
\hline$C(38)$ & $52(4)$ & $40(3)$ & $53(5)$ & $3(4)$ & $-3(4)$ & $1(3)$ \\
\hline$C(39)$ & $38(4)$ & $38(4)$ & $32(5)$ & $0(3)$ & $-9(3)$ & $5(3)$ \\
\hline$C(40)$ & $47(4)$ & $37(4)$ & $39(5)$ & $-4(3)$ & $4(3)$ & $-3(3)$ \\
\hline$C(41)$ & $42(4)$ & $57(4)$ & $66(6)$ & $-18(4)$ & $-3(4)$ & $7(3)$ \\
\hline$C(42)$ & $51(5)$ & $83(5)$ & $75(7)$ & $-16(5)$ & $-22(4)$ & $12(4)$ \\
\hline$C(43)$ & $51(5)$ & $113(7)$ & $73(7)$ & $-5(6)$ & $-16(5)$ & $-15(5)$ \\
\hline$C(44)$ & $87(6)$ & $76(6)$ & $67(7)$ & $-18(5)$ & $-3(5)$ & $-23(5)$ \\
\hline$C(45)$ & $61(5)$ & $52(4)$ & $54(6)$ & $-1(4)$ & $-6(4)$ & $-6(3)$ \\
\hline$C(46)$ & $51(4)$ & $31(3)$ & $44(5)$ & $-1(3)$ & $5(4)$ & $-5(3)$ \\
\hline $\mathrm{C}(47)$ & $65(5)$ & $54(4)$ & $54(6)$ & $-7(4)$ & $-5(4)$ & $12(4)$ \\
\hline$C(48)$ & $61(5)$ & $63(5)$ & $70(7)$ & $-18(4)$ & $9(5)$ & $4(4)$ \\
\hline$C(49)$ & $55(5)$ & $49(4)$ & $93(8)$ & $-10(5)$ & $-4(5)$ & $12(4)$ \\
\hline $\mathrm{C}(50)$ & $63(5)$ & $61(5)$ & $73(7)$ & $-14(4)$ & $-18(5)$ & $20(4)$ \\
\hline$C(51)$ & $59(5)$ & $52(4)$ & $48(5)$ & $-14(4)$ & $-5(4)$ & $1(4)$ \\
\hline$C(52)$ & $47(4)$ & $41(3)$ & $45(5)$ & $-1(3)$ & $-6(4)$ & $6(3)$ \\
\hline$C(53)$ & $83(6)$ & $41(4)$ & $87(7)$ & $-14(4)$ & $-8(5)$ & $11(4)$ \\
\hline C(54) & $62(5)$ & $49(4)$ & $57(6)$ & $3(4)$ & $1(4)$ & $-16(4)$ \\
\hline$C(55)$ & $64(5)$ & $89(5)$ & $67(7)$ & $4(5)$ & $-19(4)$ & $-23(4)$ \\
\hline$C(56)$ & $64(6)$ & $106(7)$ & $75(7)$ & $-9(6)$ & $2(5)$ & $-18(5)$ \\
\hline$C(57)$ & $64(6)$ & $95(7)$ & $111(9)$ & $-15(6)$ & $9(6)$ & $11(5)$ \\
\hline $\mathrm{C}(58)$ & $84(7)$ & $83(6)$ & $77(8)$ & $-16(5)$ & $-2(5)$ & $11(5)$ \\
\hline$C(59)$ & $63(6)$ & $89(6)$ & $70(7)$ & $-6(5)$ & $-2(5)$ & $-5(5)$ \\
\hline
\end{tabular}




\begin{tabular}{lllllll}
$\mathrm{C}(60)$ & $68(6)$ & $87(6)$ & $63(7)$ & $2(5)$ & $4(5)$ & $11(5)$ \\
$\mathrm{C}(61)$ & $78(6)$ & $76(5)$ & $46(6)$ & $3(4)$ & $9(5)$ & $-13(5)$ \\
$\mathrm{C}(62)$ & $132(8)$ & $93(6)$ & $102(9)$ & $-5(6)$ & $-7(7)$ & $-31(6)$ \\
\hline
\end{tabular}

Table 5: Hydrogen coordinates $\left(\times 10^{4}\right)$ and isotropic displacement parameters $\left(\mathrm{A}^{2} \times 10^{3}\right)$ for complex 13.

\begin{tabular}{|c|c|c|c|c|}
\hline & $\mathbf{x}$ & $\mathbf{y}$ & $\mathbf{z}$ & $\mathrm{U}(\mathbf{e q})$ \\
\hline $\mathrm{H}(1)$ & 6313 & 2783 & 398 & 62 \\
\hline $\mathrm{H}(2)$ & 7022 & 2154 & 808 & 70 \\
\hline $\mathrm{H}(3)$ & 6693 & 1713 & 1523 & 71 \\
\hline $\mathrm{H}(4)$ & 5649 & 1869 & 1799 & 60 \\
\hline $\mathrm{H}(7)$ & 4247 & 1330 & 1221 & 83 \\
\hline $\mathrm{H}(8)$ & 4491 & 259 & 1287 & 120 \\
\hline $\mathrm{H}(9)$ & 4783 & -154 & 2004 & 159 \\
\hline $\mathrm{H}(10)$ & 4818 & 472 & 2679 & 135 \\
\hline $\mathrm{H}(11)$ & 4647 & 1543 & 2612 & 96 \\
\hline $\mathrm{H}(13)$ & 5406 & 3200 & 1969 & 57 \\
\hline $\mathrm{H}(14)$ & 5799 & 3744 & 2619 & 74 \\
\hline $\mathrm{H}(15)$ & 5362 & 3636 & 3359 & 84 \\
\hline $\mathrm{H}(16)$ & 4448 & 3022 & 3463 & 90 \\
\hline $\mathrm{H}(17)$ & 4006 & 2487 & 2815 & 80 \\
\hline $\mathrm{H}(18)$ & 3201 & 2216 & 1127 & 60 \\
\hline $\mathrm{H}(19)$ & 2157 & 2167 & 850 & 63 \\
\hline $\mathrm{H}(20)$ & 1807 & 2923 & 310 & 62 \\
\hline $\mathrm{H}(21)$ & 2521 & 3676 & 39 & 54 \\
\hline $\mathrm{H}(24)$ & 2998 & 4490 & 842 & 59 \\
\hline $\mathrm{H}(25)$ & 2246 & 5165 & 1158 & 68 \\
\hline $\mathrm{H}(26)$ & 1934 & 6056 & 732 & 70 \\
\hline $\mathrm{H}(27)$ & 2384 & 6233 & -18 & 70 \\
\hline
\end{tabular}




\begin{tabular}{|c|c|c|c|}
\hline $\mathrm{H}(28)$ & 3145 & 5546 & -319 \\
\hline $\mathrm{H}(30)$ & 2584 & 4542 & -623 \\
\hline $\mathrm{H}(31)$ & 2338 & 4297 & -1398 \\
\hline $\mathrm{H}(32)$ & 3064 & 3767 & -1857 \\
\hline $\mathrm{H}(33)$ & 4072 & 3505 & -1552 \\
\hline $\mathrm{H}(34)$ & 4326 & 3765 & -774 \\
\hline $\mathrm{H}(35)$ & 5637 & 4137 & -146 \\
\hline $\mathrm{H}(36)$ & 6096 & 3836 & -853 \\
\hline $\mathrm{H}(37)$ & 5857 & 2856 & -1179 \\
\hline $\mathrm{H}(38)$ & 5145 & 2194 & -770 \\
\hline $\mathrm{H}(41)$ & 3690 & 2521 & -577 \\
\hline $\mathrm{H}(42)$ & 2992 & 2371 & -1215 \\
\hline $\mathrm{H}(43)$ & 2790 & 1367 & -1499 \\
\hline $\mathrm{H}(44)$ & 3285 & 529 & -1127 \\
\hline $\mathrm{H}(45)$ & 3960 & 658 & -479 \\
\hline $\mathrm{H}(47)$ & 5098 & 1070 & -718 \\
\hline $\mathrm{H}(48)$ & 6048 & 503 & -754 \\
\hline $\mathrm{H}(49)$ & 6636 & 314 & -70 \\
\hline $\mathrm{H}(50)$ & 6276 & 689 & 654 \\
\hline $\mathrm{H}(51)$ & 5342 & 1278 & 690 \\
\hline $\mathrm{H}(52 \mathrm{~A})$ & 4792 & 4174 & 1644 \\
\hline $\mathrm{H}(52 \mathrm{~B})$ & 4205 & 3982 & 1319 \\
\hline $\mathrm{H}(53 \mathrm{~A})$ & 4160 & 5063 & 1478 \\
\hline $\mathrm{H}(53 \mathrm{~B})$ & 4264 & 4965 & 926 \\
\hline $\mathrm{H}(53 \mathrm{C})$ & 4850 & 5158 & 1252 \\
\hline $\mathrm{H}(54 \mathrm{~A})$ & 5637 & 4666 & 834 \\
\hline $\mathrm{H}(54 \mathrm{~B})$ & 5939 & 4012 & 706 \\
\hline $\mathrm{H}(55 \mathrm{~A})$ & 6495 & 4444 & 1357 \\
\hline $\mathrm{H}(55 \mathrm{~B})$ & 6155 & 3821 & 1522 \\
\hline $\mathrm{H}(55 \mathrm{C})$ & 5849 & 4474 & 1652 \\
\hline $\mathrm{H}(56)$ & 2985 & 2028 & 2276 \\
\hline
\end{tabular}




\begin{tabular}{lllll}
$\mathrm{H}(57)$ & 2864 & 1130 & 1860 & 108 \\
$\mathrm{H}(58)$ & 1851 & 734 & 1692 & 97 \\
$\mathrm{H}(59)$ & 948 & 1277 & 1948 & 89 \\
$\mathrm{H}(60)$ & 1069 & 2182 & 2393 & 87 \\
$\mathrm{H}(62 \mathrm{~A})$ & 2596 & 2866 & 2717 & 131 \\
$\mathrm{H}(62 \mathrm{~B})$ & 1956 & 2717 & 2998 & 131 \\
$\mathrm{H}(62 \mathrm{C})$ & 1931 & 3131 & 2534 & 131 \\
\hline
\end{tabular}

